\title{
THE APPLICATION OF AUTO-TUNING PID IN ELECTRICAL AUTOMATIC CONTROL
}

\author{
Yan $\mathrm{Xi}^{1^{*}}$, Longhai $\mathrm{Fu}^{1}$ \\ 1 Yantai Vocational College, Yantai, 264000, Shandong, China. \\ Email:xiyanxiyanyt@yeah.net
}

\begin{abstract}
Objective: To explore the design and application of auto-tuning proportional integral differential (PID) in electrical automatic control and propose the application of new algorithms, thereby optimizing the auto-tuning PID controller. Methods: First, the fuzzy controller is designed, and the fuzzy algorithm is applied to the design process. Through the stepwise fuzzification and defuzzification processing of the fuzzy algorithm, the structure of the designed fuzzy controller is determined. Second, the fuzzy auto-tuning PID controller system is designed. Before designing, the control parameters are optimized and determined. The particle swarm optimization algorithm is used to select control parameters for optimization. Third, a two-dimensional fuzzy controller is used to determine the input and output variables and the membership function. Then, seven fuzzy subsets are selected to determine the number of subsets. The membership function can be divided into different shapes according to the curve shape of the function. The major membership function is the triangle membership function. Finally, the fuzzy control rules are established, and the fuzzy control algorithm and PID controller are combined to design and optimize the fuzzy auto-tuning PID controller. Results: When the operation mode is $40 \%$ load, the results obtained by applying the traditional controller and the fuzzy auto-tuning PID controller are similar, and their curve changes are consistent. With the continuous increase of load, when the operation mode becomes $60 \%, 80 \%$, and $100 \%$, the control curve of fuzzy auto-tuning controller is more stable than traditional control. In addition, the response time of the system also gets faster. During the control process, no obvious fluctuations occur. However, the overshoot of the response system of the traditional controller increases with the increase of the load. When the load is $100 \%$, the obvious oscillation even occurs. Comparing the two curves under different loads, the fuzzy auto-tuning is more stable and has less change than the control curve of the traditional controller. Conclusion: The designed fuzzy autotuning PID control performance has excellent stability and controllability. It has important application value in electrical system control, which can be widely used in automatic control of electrical systems.
\end{abstract}

Keywords: Auto-Tuning PID; Electrical; Automatic Control; Fuzzy Algorithm.

\section{Introduction}

Motion control system is an electric drive control system composed of mechanical motion driving equipment. Guided by the theory of automatic control, it takes motor as the control object, the controller as the core, and the power conversion device and power electronics as the execution mechanism [1-3].

The system controls the speed, rotation angle and torque of the motor, converts electrical energy into mechanical energy, and realizes the motion requirements of mechanical devices [4].

In the information age, high-tech flows to traditional industries, causing the profound changes in the latter. As one of the traditional industries, the machinery industry has undergone qualitative changes in the structures of product and production system under the impact of the technological revolution. Microelectronic technology and microcomputer technology make information and intelligence combined with mechanical devices and power equipment, and promote general motion control technology to become an important branch of automation technology [5].

Motion control is no longer limited to the traditional machinery industry. It is widely used in machine tools, automobiles, meters, household appliances, light industrial machinery, textile machinery, packaging machinery, printing machinery, metallurgical machinery, chemical machinery, industrial robots, and intelligent robots, which makes new progress annually.

Traditional proportional integral differential (PID) control is widely used in various industrial control processes due to its advantages, such as 
simple structure, easy implementation, elimination of residuals, and rapid action.

Currently, PID control still accounts for $84 \%$ of the control systems worldwide [6].

PID control also occupies a dominant position in control systems such as boilers and steam turbines in thermal power plants.

However, in the control system of boilers, steam turbines, and other equipment in thermal power plants, since these controlled objects are timevarying, non-linear, and uncertain, the effect of conventional PID control cannot meet the requirements of system control accuracy [7].

The reason for this problem is that the premise that PID control can be implemented accurately and effectively is to first establish an accurate mathematical model of the controlled object.

When the controlled object frequently changes during operation, the PID controller cannot achieve the desired control effect.

Also, in the production process, the models of the controlled object are mostly non-linear systems, which have the characteristics of lag and timevariant; some models even cannot be established [8].

To improve the control performance of PID controllers, engineering technicians are exploring the combination with some new control methods to make the control effect closer to the ideal $[9,10]$.

In this study, the self-tuning PID control system in the electrical system is designed.

First, the fuzzy controller is designed by using the fuzzy algorithm.

Through the fuzzification and defuzzification processing of the fuzzy algorithm, the structure of the designed fuzzy controller is determined.

Second, the fuzzy controller and auto-tuning PID controller are combined.

The particle swarm optimization algorithm is used to select control parameters for optimization. Third, a two-dimensional fuzzy controller is used to determine the input and output variables and the membership function. Seven fuzzy subsets are selected to determine the number of subsets.

Finally, the fuzzy control rules for three control parameters are established.

The previously designed fuzzy control system is integrated into the auto-tuning PID control system.

A simulation experiment of the optimized control system is conducted on the bed-temperature electrical control system of circulating fluidized bed in MATLAB to verify the effectiveness and superiority of the system.

The experimental results show that the system designed in this study has a smoother control curve than traditional control systems.

Without much fluctuation, the designed system has a faster response speed and reduced overshoot. As the operation load continues to increase, its superiority also gets more apparent.
Therefore, the fuzzy self-tuning PID control system designed in this study has certain effectiveness, stability, controllability, and robustness, which can well-avoid the disadvantages of traditional control systems.

Meanwhile, the designed system can effectively achieve the controlling purposes of the device, which can be widely used in electrical automatic control systems.

\section{Methods \\ 2.1 Design of fuzzy controller}

Fuzzification maps the range of variation to the corresponding domain, transforms the input data in the domain into corresponding linguistic variables, and forms a fuzzy set [11]. In other words, the sensor converts the relevant physical quantity of the controlled object into electric quantity.

If the output quantity is a continuous analog quantity, it is converted again by the A/D converter converts into a digital quantity as the input measurement value of the computer; then, the measured value is standardized.

Therefore, the exact input is converted into the value of a fuzzy variable represented by the membership function, and A and B are the fuzzy sets on the domain.

$$
\begin{aligned}
& A=\left\{a_{1}, a_{2}, \ldots, a_{m}\right\} \\
& B=\left\{b_{1}, b_{2}, \ldots, b_{m}\right\}
\end{aligned}
$$

If the union of $A$ and $B$ is $A \cup B$, the intersection of $\mathrm{A}$ and $\mathrm{B}$ is $\mathrm{A} \cap \mathrm{B}$, and the complement set of $\mathrm{A}$ is $\bar{A}$, then:

$$
\begin{gathered}
\mu_{(A \mathrm{U} B)}(\mathrm{x})=\max \left(\mu_{\mathrm{A}}(\mathrm{x}), \mu_{B}(\mathrm{x})\right), \\
\forall \mathrm{x} \in \mathrm{U} \\
\mu_{(A \mathrm{U} B)}(\mathrm{x})=\min \left(\mu_{\mathrm{A}}(\mathrm{x}), \mu_{B}(\mathrm{x})\right), \forall \mathrm{x} \in \mathrm{U} \\
\mu_{(\bar{A})}(\mathrm{x})=1-\mu_{(A)}(\mathrm{x}), \forall \mathrm{x} \in \mathrm{U}
\end{gathered}
$$

The fuzzy relation between A and B is defined as a fuzzy subset of the Cartesian set $A \times B$. If $a$ membership function is used to represent a fuzzy subset, the fuzzy relation can be represented by a matrix, i.e.:

$$
R_{A \times B}=\left[\begin{array}{cccc}
\mu_{11} & \mu_{12} & \ldots & \mu_{1 n} \\
\mu_{21} & \mu_{22} & \ldots & \mu_{2 n} \\
\cdot & \cdot & \cdot & \cdot \\
\cdot & \cdot & \cdot & \cdot \\
\cdot & \cdot & . & \cdot \\
\mu_{m 1} & \mu_{m 2} & \ldots & \mu_{m 3}
\end{array}\right]
$$




$$
\text { If } X=\left\{x_{1} / a_{1}, x_{2} / a_{2}, \ldots, x_{m} / a_{m}\right\} \quad \text { is a }
$$

membership function on domain $\mathrm{A}$, which is simply represented by vector $X=\left\{x_{1} / a_{1}, x_{2} / a_{2}, \ldots, x_{m} / a_{m}\right\}$. Then, the weigh vector is:

$$
\begin{aligned}
& Y=\left(y_{1}, y_{2}, \ldots, y_{n}\right) \\
& Y=X \bullet R_{A \times B}
\end{aligned}
$$

It is the result of the fuzzy transformation of $\mathrm{X}$, which represents a membership function on domain B:

$$
\begin{aligned}
& Y=\left\{y_{1} b_{1}, y_{2} b_{2}, \ldots, y_{n} b_{n}\right\} \\
& \mathrm{y}_{1}=\theta_{k=1}^{m} \mu_{k i} \bullet x_{k}(i=1,2, \ldots, \mathrm{n})
\end{aligned}
$$

Afterward, the step of inferring the fuzzy control rule by using the detected input amount as a condition in the fuzzy control rule can be completed. In addition, the module that can accomplish the step is called a state interface or a fuzzification interface.

The accurate measurement of the input quantity is the most critical process.

The input quantity mainly includes the input of the external system and the output of the system.

The fuzzy output is not directly controllable to the executing component. It is necessary to determine the most representative value as the output control amount in the determined output range.

This method is called deblurring judgment.

Different algorithms are obtained from different angles and aspects to determine the key values.

The module is called the deblurring interface or control interface.

The function of deblurring module is to convert the amount of blur obtained from the previous legend into an accurate quantity for actual control.

After obtaining the magnitude, the logarithmic mode is converted to obtain an accurate analog signal and transmitted to the actuator to control the controlled object.

Through the above basic introduction to the fuzzy control theory, the fuzzy control algorithm is summarized, which is divided into the following four steps.

First, the output value of the system obtained at the beginning is calculated to obtain the input variable of the selected system.

Then, it is divided into fuzzy quantities.

Afterward, according to the fuzzy quantity of the input variable and the fuzzy control rule, the inference synthesis is the fuzzy control system rule to calculate the control quantity.
Finally, the deblurring process is used to obtain the precise control quantity.

In the process of defuzzification, the major principle is to convert the fuzzy amount into a hierarchical amount, which is then converted into an exact amount.

In addition, it can be transformed into a hierarchical quantity by applying the maximum membership degree method and the weighted average judgment method.

The principle of the maximum membership degree method is to find the subset with the largest degree of membership in the fuzzy subset of the fuzzy controller, and define its elements as $u_{i}$. In the controller, if the continuous elements have the largest degree of membership, the average value of the elements is calculated, and the average value is used as the output value of the control amount.

The principle of applying the maximum membership degree method to defuzzify is simple, but its resource information is limited.

It can only exclude some elements of small membership degree, but cannot reflect the effect of each element on the control result.

The major property of weighted average decision method is a selection method based on weight coefficients.

The unique attributes of the system will determine the weight coefficient.

The weight coefficient is assumed as $\mathrm{k}_{\mathrm{i}}=(\mathrm{i}=1$, $2, \ldots, \mathrm{m}$ ); then, the equation for calculating the fuzzy judgment output is as follows:

$$
U=\frac{\sum_{i=1}^{m} k_{i} u_{i}}{\sum_{i=1}^{m} k_{i}}
$$

The exact amount can be obtained based on the fuzzy judgment output and the travel of the actuator. If the maximum travel that the actuator can accept within a limited number of times is $\pm u_{m} \%$, the output field of the controller can be set to:

$$
u=\left[-u_{m}, u_{m}\right]
$$

Therefore, the domain of rank can be set as:

$$
U=[-m, m]
$$

The equation for calculating the output of the controller is as follows:

$$
u=\frac{u_{m}}{m} U=K_{u} U
$$

Where: Ku represents a scale factor.

The following figure shows the design process of the fuzzy controller: 


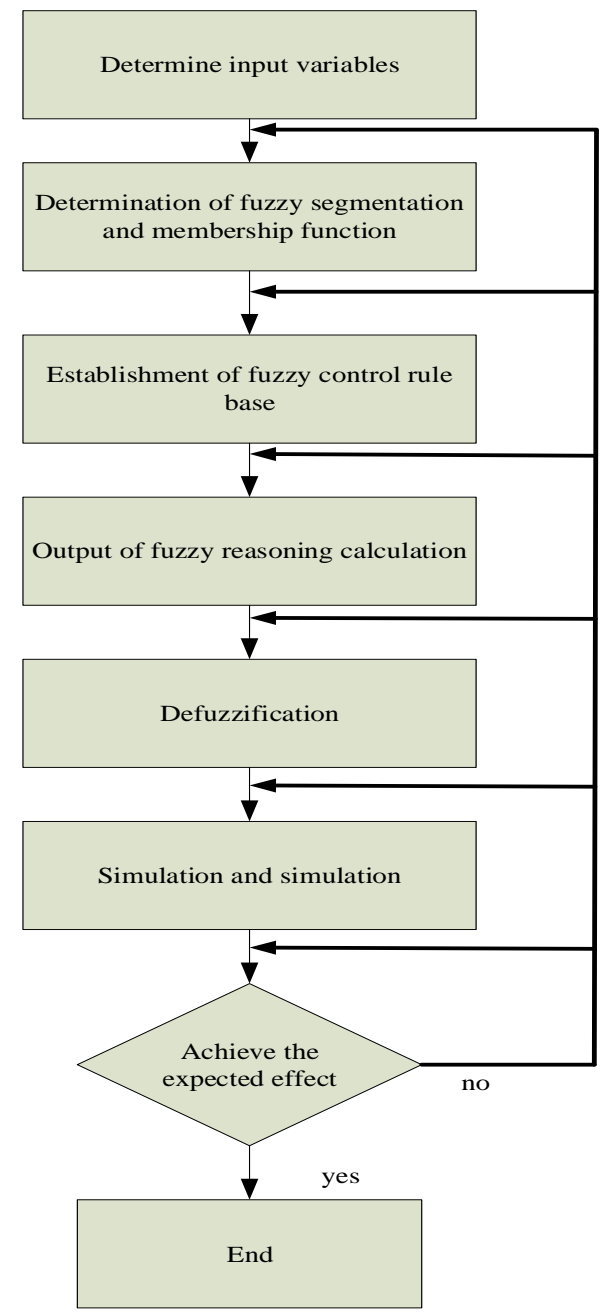

Figure 1: Design process of fuzzy controller

\subsection{Design of fuzzy auto-tuning PID controller system}

Before designing, the correct PID parameters are selected. Generally, there are various methods for selecting parameters, such as genetic algorithm, ant colony optimization algorithm, and particle swarm algorithm. This study uses the particle swarm algorithm to perform parameter selection. Compared with other algorithms, this algorithm has simple operation and calculation, which makes it easier to be implemented [12].

First, the three PID parameters are converted into a collection in the particle swarm algorithm. Since each particle has a specific direction and speed of movement, when the state of its movement changes, the fitness function is applied to calculate the particle fitness. Through this method, the particle swarm with the best fitness is found, which is considered as the optimal PID parameter.

Figure 2 shows the optimized structure of the PID parameters based on the particle swarm algorithm.

As shown in the figure, this controller is composed of two parts.

The first part is the PID control line to control the controlled object.

The particle swarm algorithm can be used to effectively adjust the parameters.

The second part is based on the real-time operation of the system.

In this case, the particle swarm algorithm is used to optimize the parameters to adapt to the operation of the system, thereby improving the control performance of the optimized system.

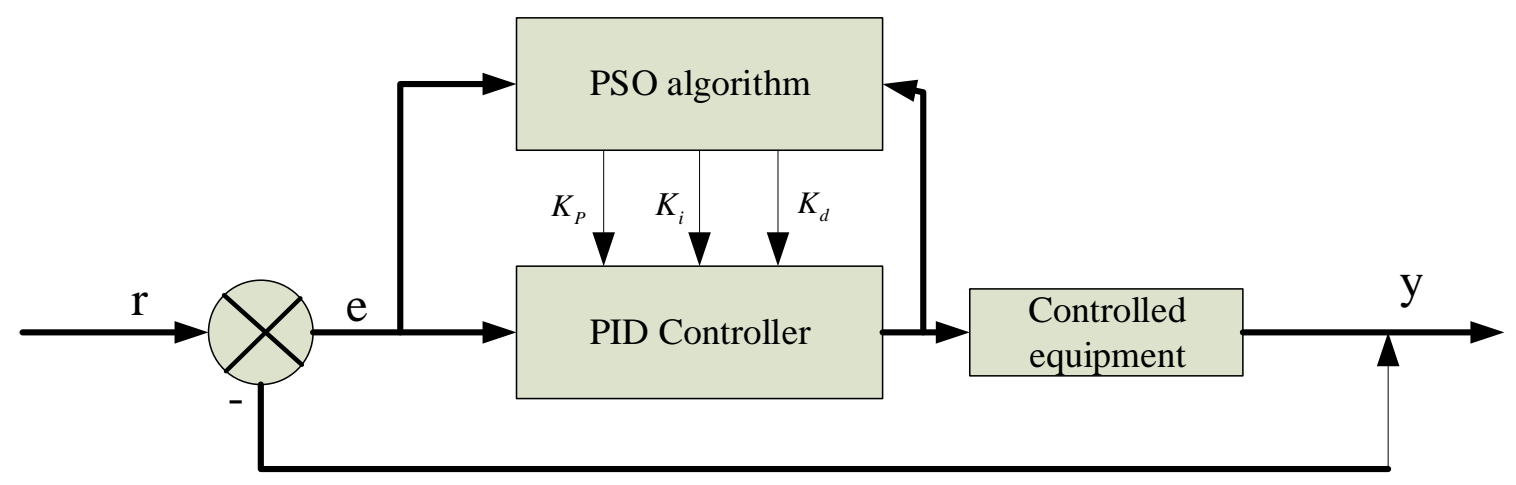

Figure 2: The optimized structure of PID parameters based on particle swarm algorithm

The second is to determine the input and output variables and membership function of the fuzzy controller. This study uses the two-dimensional fuzzy controller. The deviation of the input value is set to e and the deviation change rate is set to ec. The fuzzy language variable of the input value deviation is represented by $\mathrm{E}$, the fuzzy language variable of the deviation change rate is represented by EC, and the parameter correction amount of the output PID controller is respectively represented by $\Delta k_{p}, \Delta k_{i}$ and $\Delta \mathrm{k}_{\mathrm{d}}$. It is necessary to fuzzify and defuzzify the exact amount and the fuzzy amount to obtain the results of fuzzy reasoning and fuzzy output values.

The value range of the deviation e of the input value is assumed as $\left[-\left|e_{\max }\right|,\left|e_{\max }\right|\right]$; then, the value range of the deviation change rate can be set to $\left[-\left|e c_{\max }\right|,\left|e c_{\max }\right|\right]$, and the value range of the input variable $\mathrm{u}$ is $[-u, u]$. Therefore, the corresponding 
value range of the fuzzy language variable $\mathrm{E}$ is $\left\{-n_{e},-n_{e}+1, \ldots, 0, \ldots n_{e}-1, n_{e}\right\}$.

Therefore, in this study, the quantization factor and scale factor can be calculated according to the basic fuzzy domain and the maximum value of the fuzzy subset domain of the variable.

$$
k=\frac{n}{m}
$$

Where: $\mathrm{n}$ represents the minimum value of the fuzzy subset domain, and $m$ represents the maximum value of the fuzzy subset domain. The equation for calculating the quantization factor of the deviation is as follows:

$$
k_{e}=\frac{n_{e}}{e_{\max }}
$$

The equation for calculating the deviation change rate and the quantization factor of the output $\mathrm{u}$ is as follows:

$$
\begin{aligned}
& k_{e c}=\frac{n_{e}}{e c_{\max }} \\
& k_{u}=\frac{n_{u}}{u}
\end{aligned}
$$

Then, seven fuzzy subsets are selected to determine the number of subsets. The membership function can be divided into different shapes according to the curve shape of the function, such as triangles, trapezoids, and bells. This study uses the triangle membership function and the normal membership function, the equation is as follows:

$$
\text { triangle }(x, a, b, c)=\left\{\begin{array}{c}
0, x \leq a \\
\frac{x-a}{b-a}, a \leq x \leq b \\
\frac{c-x}{c-b}, b \leq x \leq c \\
0, x \geq c
\end{array}\right.
$$

Where: the relation among $a, b$ and (19) $a \leq b \leq c$. Figure 3 is a function curve of triangle membership:

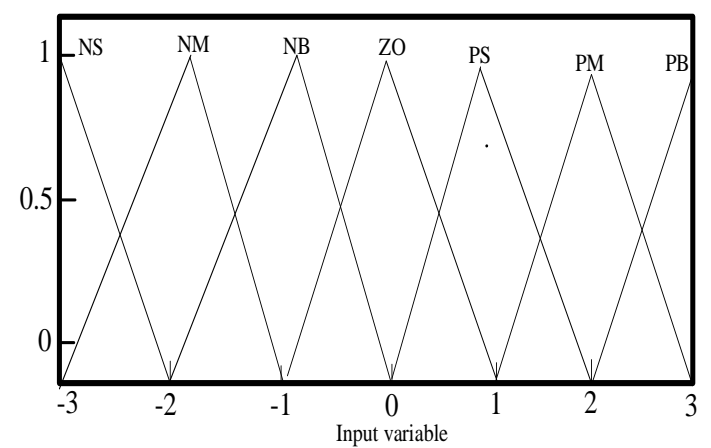

Figure 3: The function curve of triangle membership

The equation for the normal membership function is as follows:

$$
\operatorname{Gass}(x, a, b)=e^{\frac{1}{2}\left(\frac{x-a}{b}\right)^{2}}
$$

Where: a represents the middle value of the normal table membership function, and $b$ represents the width of the function.

Under normal circumstances, conventional PID controllers mainly rely on accurate mathematical models. However, in practical applications, many control systems have more complex structures, with non-linear and time-varying features [13].

In the actual controlling process, all sudden situations that occur will affect the accuracy and stability of the system and reduce the control effect of the system. Therefore, it is necessary to combine fuzzy control and auto-tuning PID.

The composite control has no strict requirements on the mathematical model of the controlled object. Only the appropriate fuzzy rules need to be established in the knowledge base, and the system parameters are appropriately selected.

These parameters mainly include PID initial parameters and evaluation indicators. The fuzzy controller will constantly adjust and adapts to changing conditions of the system.

Then, the fuzzy control rules are used for correct reasoning and decision-making, as shown in Figure 4 , the structure diagram of the fuzzy auto-tuning PID controller:

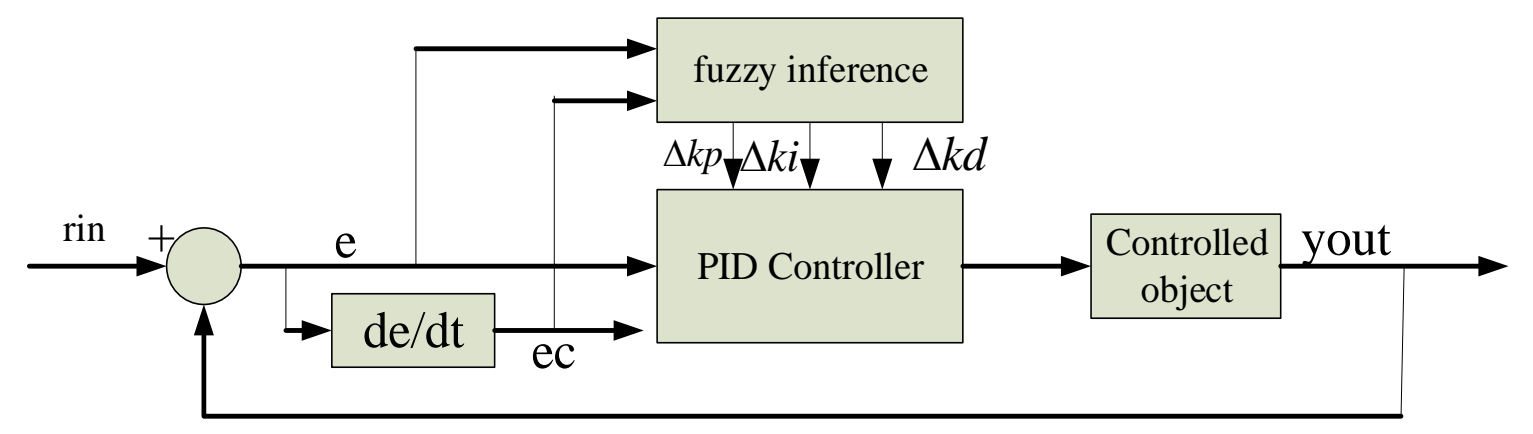

Figure 4: The structure of PID controller 


\subsection{Establishment of fuzzy control rules}

The controllability will be directly affected by the fuzzy control rules. Therefore, it is vital to establish appropriate and correct fuzzy control rules.

This study is mainly based on the traditional PID controller to adjust and optimize the parameters of the fuzzy auto-tuning PID controller system, as well as constantly optimizing its parameters according to the actual situation. Therefore, it can effectively improve the control performance of the system and optimize the control effect. It is very important to determine the rules of fuzzy control, whose major purpose is the fuzzy control of PID parameters.

The first is the establishment of a control rule for the proportional coefficient KP. To make the system respond quickly at the beginning of control, the KP value should be selected to be the largest. During the middle of the control, the system needs to maintain stable. Only a small deviation is acceptable.

Therefore, there are certain requirements for selecting the KP value, which is required to be relatively large. In the later control process, the parameter value needs to be further increased to a larger level, so that the system can be effectively maintained at a high accuracy level.

The second is the establishment of Ki fuzzy rule of integration coefficient.

The major purpose of this integration coefficient is to eliminate the steady-state error caused by the system. At this time, there is a certain relationship between this integration coefficient and the speed of eliminating the static difference. It is proportional; however, if it exceeds a certain range, the negative phenomenon of increasing the overshoot will occur and even the integral to saturate.

In addition, it will also prolong the system response time, which will negatively affect the system performance.

The third is the establishment of the fuzzy rule of the differential coefficient $\mathrm{Kd}$.

The major purpose of this differential coefficient is to optimize and improve the overall performance of the system, to prevent the system from deviating, and to change in any direction.

At the beginning of control, this differential coefficient should be increased to reduce the overshoot of the adjustment process.

In the middle of the controlling process, the minimum value of the differential coefficient should be selected, thereby reducing the negative influence of the differential effect on the system change.

In the final stage of the control process, this differential coefficient needs to be reduced to achieve the purpose of reducing the hindering change brought by the differential coefficient.

\section{Results}

At present, many electrical control systems still use traditional PID controllers. Although they can meet the basic control requirements under normal circumstances, they cannot handle complex control systems with large inertia and large delays.

The application of traditional control systems cannot meet the control requirements and objectives, which needs to be adjusted by manual operation. In this study, the control system designed and researched is applied to electrical automatic control in real-time.

This study uses a 300 MW circulating fluidized bed in a thermal power plant as an example to compare the traditional PID controller with the designed PID controller.

The simulation experiments are performed separately, and the effectiveness of the designed system is verified by comparing the control effects of the two controllers.

There are different models for different operation modes, and their transfer function models are also different.

There are four different operation modes in the simulation experiment, which are 100\% load, 80\% load, $60 \%$ load, and $40 \%$ load.

According to the different transfer function of each operation mode, the parameter range of the controller is estimated and calculated.

The range of KP is between 0.1 and 0.2 , the range of $\mathrm{Ki}$ is 0.0003 to 0.0005 , and the range of $\mathrm{Kd}$ is between 0.3 and 0.7 .

Figures 5-8 show the system dynamic response comparison curve for the traditional controller and the designed fuzzy auto-tuning PID controller under different operation modes.

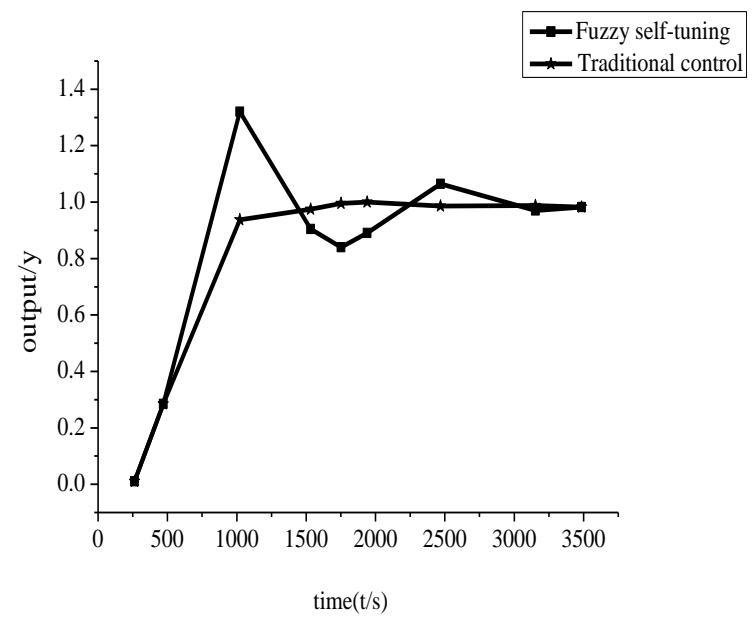

Figure 5: Step disturbance results at 100\% load 


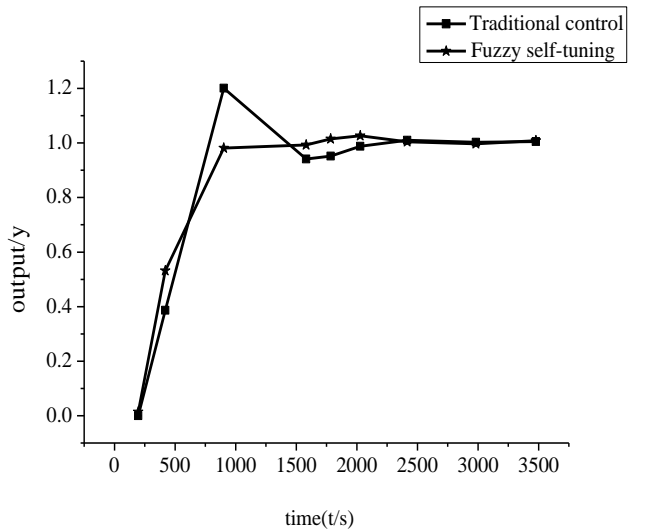

Figure 6: Step disturbance results at $80 \%$ load

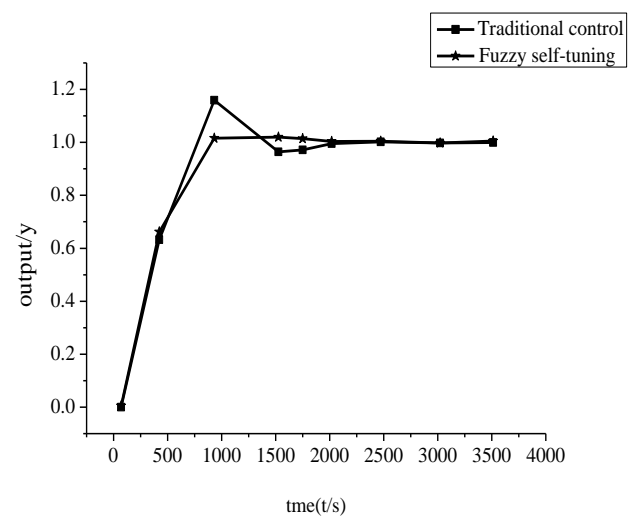

Figure 7: Step disturbance results at 60\% load

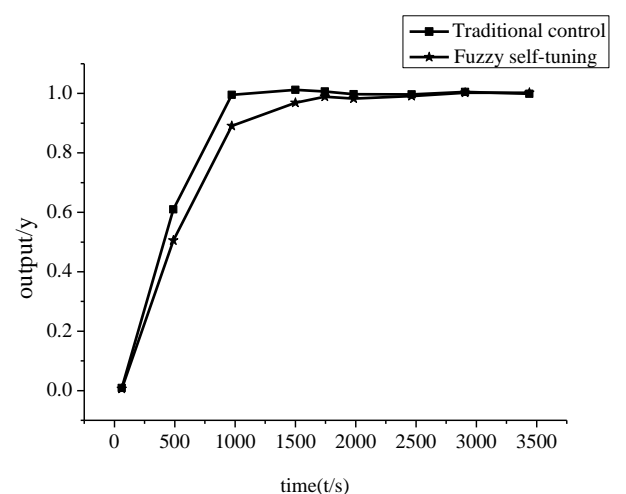

Figure 8: Step disturbance results at 40\% load

According to the above figure, when the working condition is $40 \%$ load condition, the results obtained by applying the traditional controller and the fuzzy self-tuning PID controller designed in this study are not very different.

The curve changes are the same, but in terms of control response, the overshoot of the fuzzy selftuning controller is smaller, which requires less adjustment time than the traditional controller, and has better response to system dynamics. With the continuous increase of working load, when it gets $60 \%, 80 \%$, and $100 \%$, the performance of the fuzzy auto-tuning system designed in this study is more superior. The control curve of the fuzzy auto-tuning controller is more stable than traditional controllers.
In addition, the response time of the control system also gets faster. During the controlling process, there is no obvious fluctuation. However, the overshoot of the response system of the traditional controller increases with the increase of the load. When the load is $100 \%$, the obvious oscillation even occurs.

Comparing the two curves under different loads, the fuzzy auto-tuning is more stable and has less change than the control curve of the traditional controller.

Therefore, the fuzzy auto-tuning PID control system designed in this study has good stability and robustness. It can adapt to different working loads, has superior control performance, and can be appropriately adjusted according to different control conditions to adapt to changes in the system.

\section{Discussion}

With the development of the times, the objects of automatic control become increasingly complex.

However, the performance requirements of control systems are constantly increasing.

A complex system is mainly manifested by the strong coupling between multiple input and output variables of the system, changes in the parameters of the controlled objects, and the uncertainty and serious non-linearity of the system structure [14, 15].

Fuzzy control is based on the theory of fuzzy mathematics. It is an intelligent control method based on rule judgment. Its theoretical method and implementation technology are quite different from traditional control theories and technologies.

Although fuzzy control is a relatively new type of intelligent control, it must also follow certain laws and rules, i.e., fuzzy control should also have a corresponding control mechanism and action principle [16].

The core of practical application of fuzzy control theory mainly lies in the design and implementation of fuzzy controller, i.e., the control mechanism of fuzzy control is mainly reflected by fuzzy controller.

In this study, the self-tuning PID control system in the electrical system is designed.

The fuzzy algorithm is used in the optimization of fuzzy controller design, as well as the defuzzification and calculation of fuzzy algorithm.

Meanwhile, the particle swarm optimization algorithm is used to select and optimize the control parameters. A two-dimensional fuzzy controller is used to determine the input and output variables and the membership function.

Finally, simulation experiments of the optimized control system are conducted on the bedtemperature electrical control system of circulating fluidized bed in MATLAB to verify the effectiveness and superiority of the system. 
The research results show that with the continuous increase of working load, when it gets $60 \%, 80 \%$, and $100 \%$, the control curve of the fuzzy auto-tuning controller is more stable than traditional controllers. In addition, the response time of the control system also gets faster. During the controlling process, there is no obvious fluctuation.

According to the experimental results, the fuzzy auto-tuning PID control system designed in this study has certain effectiveness and superiority, which can well control the electrical automatic control system and has better performance than the traditional controllers.

It is more robust and has a faster response speed. In addition, the designed system can also adapt to different working conditions of the system and realtime changing working conditions, and has super stability.

\section{Conclusions}

In this study, a fuzzy controller is designed by applying the fuzzy algorithm. The fuzzy controller is combined with an auto-tuning PID controller.

The fuzzy algorithm is fuzzified and defuzzified, and the controller parameters are appropriately selected and optimized. The fuzzy control rules for controller parameters are established, and the dimensions of the input variables of the fuzzy controller are determined.

Finally, through simulation experiments, the effects of input variable dimensions, adjustment factors, and disturbance factors on the fuzzy controller are analyzed and discussed. Through experiments, the superiority, effectiveness, stability and controller of the fuzzy auto-tuning PID control system designed in this study have been verified, which has an excellent application value in electrical automatic control. However, the structure of the fuzzy controller designed in this study is simple. It does not consider the various complex and difficult problems and situations in actual operations. Instead, it just applies simple operation and application in the simulation system. In the subsequent study, complicated issues to the actual scenes should be considered.

\section{References}

[1] Hélio F., Freire, Paulo B. (2015) de Moura Oliveira, Eduardo J. Solteiro Pires, et al. Manyobjective PSO PID controller tuning. Lecture Notes in Electrical Engineering, 321, 183-192.

[2] Kazuhiko T., Yuka S., Masafumi H. (2015) Selftuning PID controller using quantum neural network with qubit-inspired neurons. International Journal of Hybrid Intelligent Systems, 12(1), 41-52.

[3] Pan C., Zhang R., Chen L, et al. (2017) Research on Variable Current Regenerative Braking Control Strategy Based on Radial Basis Function Neural Network Tuning PID Control. Journal of
Computational \& Theoretical Nanoscience, 14(1), 468-476.

[4] Wang X. K., Wang L. (2016) Design and Research based on Fuzzy PID-parameters Self-tuning Controller with MATLAB. New Technology \& New Process, 996-999.

[5] Camcioğlu Ş., Özyurt B., Doğan İ. C, et al. (2017) Application of response surface methodology as a New PID tuning method in an electrocoagulation process control case. Water Science \& Technology, 76(12), 2017506.

[6] Zhang M., Borja P., Ortega R, et al. (2017) PID Passivity-Based Control of Port-Hamiltonian Systems. IEEE Transactions on Automatic Control, 99, 1-1.

[7] Gu Q., Zheng F. L., Liu B. B, et al. (2015) Application of a New Smith Fuzzy PID in Electric Boiler Temperature Control System. Applied Mechanics \& Materials, 727-728:633-636.

[8] Romero J. G., Ortega R., Donaire A. (2016) Energy Shaping of Mechanical Systems via PID Control and Extension to Constant Speed Tracking. IEEE Transactions on Automatic Control, 61(11), 1-1.

[9] Sahu B. K., Pati S., Mohanty P. K, et al. (2015) Teaching-learning based optimization algorithm based fuzzy-PID controller for automatic generation control of multi-area power system. Applied Soft Computing, 27, 240-249.

[10] Madasu S. D., Kumar M. L. S. S., Singh A. K. (2016) A flower pollination algorithm based automatic generation control of interconnected power system. Ain Shams Engineering Journal, $9(4)$.

[11] Çelik E. (2018) Incorporation of stochastic fractal search algorithm into efficient design of PID controller for an automatic voltage regulator system. Neural Computing \& Applications, 30(6), $1-12$.

[12] Li M., Zhen Z. Y., Gong H. J, et al. (2017) Optimal Preview Control for Automatic Carrier Landing System of Carrier-Based Aircraft with Air Wake. Transactions of Nanjing University of Aeronautics and Astronautics, 34(6), 659-668.

[13] Yang S. M., Lin K. W. (2015) Automatic Control Loop Tuning for Permanent Magnet AC Servo Motor Drives. IEEE Transactions on Industrial Electronics, 63(3), 1-1.

[14] Bazanella A. S., Pereira L. F. A, Parraga A. (2016) A New Method for PID Tuning Including Plants Without Ultimate Frequency. IEEE Transactions on Control Systems Technology, 25(2), 1-8.

[15] Haibo L., Hongyan L. (2016) Control Model of Throttle Back Pressure of Managed Pressure Drilling. Journal of Computational \& Theoretical Nanoscience, 13(10), 7603-7609.

[16] Ma X. Y. (2017) Fuzzy PID temperature control system for DFB laser based on adaptive genetic algorithm. Shenyang Gongye Daxue Xuebao/journal of Shenyang University of Technology, 39(4), 454-458. 